\title{
Screening the physical properties of a series of water-in-salt electrolytes using computer simulations
}

Trinidad Mendez-Morales, ${ }^{1,2,3}$ Zhujie Li, ${ }^{1,2,3}$ and Mathieu Salanne ${ }^{1,2,3,4, \text { a) }}$

1) Maison de la Simulation CEA, CNRS, Université Paris-Sud, UVSQ, Université Paris-Saclay, F-91191 Gif-sur-Yvette, France

2) Sorbonne Université, CNRS, Physico-Chimie des Électrolytes et Nanosystèmes Interfaciaux, F-75005 Paris, France

3) Réseau sur le Stockage Électrochimique de l'Énergie (RS2E), FR CNRS 3459, Amiens, France

4) Institut Universitaire de France (IUF), 75231 Paris Cedex 05, France

Water-in-salts form a new family of electrolytes with properties distinct from the ones of conventional aqueous systems and ionic liquids. They are currently investigated for Li-ion batteries and supercapacitors applications, but to date most of the focus was put on the system based on the LiTFSI salt. Here we study the structure and the dynamics of a series of water-in-salts with different anions. They have a similar parent structure but they vary systematically through their symmetric/asymmetric feature and the length of the fluorocarbonated chains. The simulations allow to determine their tendency to nanosegregate, as well as their transport properties (viscosity, ionic conductivity, diffusion coefficients) and the amount of free water, providing useful data for potential applications in energy storage devices.

a) Electronic mail: mathieu.salanne@sorbonne-universite.fr 


\section{INTRODUCTION}

Lithium-ion batteries (LIBs) are currently the most important energy-storage devices. They are employed in a wide range of applications due to their properties ${ }^{1,2}$. They generally involve organic electrolytes, which allows to reach high voltages and thus increases the energy density of the devices. However, some concerns remain associated with their cost, safety and environmental impact ${ }^{3,4}$. Aqueous electrolytes can be an alternative to solve these problems, but the use of water as solvent results in a much narrower electrochemical stability window ${ }^{5-7}$.

Recently, a new class of water-in-salt (WiS) electrolytes was reported by Suo et al. ${ }^{8}$ to expand the electrochemical stability window up to nearly $3 \mathrm{~V}$ by using aqueous solutions of lithium bis[(trifluoromethyl)sulfonyl]imide (LiTFSI) with a molality of $21 \mathrm{~m}(\mathrm{~mol} / \mathrm{kg})$. They are thus a subclass of solvent-in-salt ${ }^{9}$ electrolytes, which are defined as systems in which the salt over solvent mass and volume ratios are greater than 1. In such superconcentrated electrolytes the increased stability window is due to several effects ${ }^{10}$ : Firstly, water molecules display a specific speciation, since most of them belong to the lithium ion solvation shells, thus leading to low fractions of free water molecules. This reduces the concentration of water at the positive interface, which mainly contains TFSI $^{-}$anions ${ }^{8,11}$. However, the main reason for the extended voltage window is the formation of a solid electrolyte interface (SEI) layer that mainly consists of LiF as a result of the electrochemical decomposition of the TFSI anion ${ }^{12-14}$. Although most of the studies of WiS electrolytes were performed using the LiTFSI salt ${ }^{15-21}$, the WiS concept was extended to other metallic ions such as potassium ${ }^{22}$, sodium $^{23-26}$ and zinc-based ${ }^{27}$ electrolytes.

In parallel, the chemistry of Li-ion based WiS electrolyte family was also explored further. For example, since the concentration of LiTFSI in the WiS electrolyte is limited by the solubility limit, it was proposed to use a water-in-bisalt electrolyte, that is, to use LiTFSI as a parent hydrate salt to dissolve another lithium salt (LiOTf), to obtain a higher efficiency ${ }^{28,29}$. On the contrary, the WiS electrolytes form biphasic systems when they are mixed with simpler solutions such as highly concentrated aqueous $\mathrm{LiCl}^{30}$, which was exploited to develop a dual battery involving lithium ions together with halogen conversion-intercalation ${ }^{31}$. A mixture of lithium and potassium acetate-based WiS was also recently proposed as a greener and lower-cost alternative to TFSI-based systems ${ }^{32}$, showing further the versatility of this 
family of electrolytes.<smiles>O=S(=O)([O-])C(F)(F)F</smiles>

TF<smiles>O=S(=O)(NS(=O)(=O)C(F)(F)F)C(F)(F)F</smiles>

TFSI<smiles>O=[Si](=O)(F)NS(=O)(=O)F</smiles>

FSI<smiles>N#CNS(=O)(=O)C(F)(F)F</smiles>

TFSAM<smiles>O=C(NS(=O)(=O)C(F)(F)F)C(F)(F)F</smiles>

TSAC

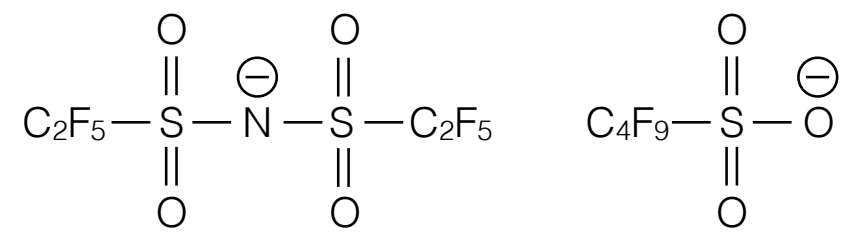

BETI

NF

FIG. 1. Chemical formulae of the various anions investigated in this work. The full names of the molecules are trifluoromethanesulfonate (TF), bis(fluorosulfonyl)imide (FSI), 2,2,2-trifluoromethylsulfonyl-N-cyanoamide (TFSAM), 2,2,2-trifluoro-N-(trifluoromethylsulfonyl) acetamide (TSAC), bis[(trifluoromethyl)sulfonyl]imide (TFSI), bis[(pentafluoroethyl)sulfonyl]imide (BETI) and nonafluorobutanesulfonate (NF).

In practice, it could be possible to develop WiS electrolytes with a large number of different anions. Here we show that molecular dynamics simulations provide a convenient framework for studying their physical properties. We studied seven different anions, including five which were imide-based, namely bis[(trifluoromethyl)sulfonyl]imide (TFSI), bis(fluorosulfonyl)imide (FSI), bis[(pentafluoroethyl)sulfonyl]imide (BETI), 2,2,2trifluoromethylsulfonyl-N-cyanoamide (TFSAM) and 2,2,2-trifluoro-N-(trifluoromethylsulfonyl) acetamide (TSAC), and two sulfonate anions, trifluoromethanesulfonate (TF) and nonafluorobutanesulfonate (NF). Their chemical formulae are provided on Figure 1. We first study the structure at a large molality of $15 \mathrm{~mol} \mathrm{~kg}^{-1}$. One of the systems shows a tendency to phase separation, so that we discard it from the other analysis. We then compare the conductivity, the viscosity of the liquids as well as the individual diffusion coefficients of all the species. Comparing these transport properties allows to rank the various anionic species for applications. Finally, we determine the amount of "free" water in the various systems since this quantity is known to influence the reactivity at the electrochemical interface. 


\begin{tabular}{cc}
\hline Lithium salt & Density $\left(\mathbf{k g} / \mathbf{m}^{\mathbf{3}}\right)$ \\
\hline LiTFSI & 1692.577 \\
LiFSI & 1631.462 \\
LiBETI & 1751.465 \\
LiTF & 1570.122 \\
LiNF & 1770.777 \\
LiTFSAM & 1533.237 \\
LiTSAC & 1680.515 \\
\hline
\end{tabular}

TABLE I. Densities obtained for the seven WiS electrolytes at a concentration of $15 \mathrm{~m}$ and room temperature. The experimental density of a LiTFSI- $\mathrm{H}_{2} \mathrm{O}$ mixture under the same conditions is $1673 \mathrm{~kg} / \mathrm{m}^{3}$, which corresponds to an error of $1.2 \%$.

\section{SIMULATION DETAILS}

MD simulations of WiS electrolytes were carried out using the LAMMPS package ${ }^{33}$. All cubic simulation boxes contained 473 water molecules and 128 salt ion pairs that were randomly distributed using $\mathrm{Packmol}^{34}$. The SPC/E water model was used in these simulations $^{35}$, whereas the parameterization of the anions was made in the framework of the CL\& $\mathrm{P}^{36}$ force field for ionic liquids, for which we employed the set of parameters reported in Ref. 37. Lithium cations were modelled as a single site whose Lennard-Jones (LJ) parameters are $\epsilon=6.25 \mathrm{kcal} / \mathrm{mol}$ and $\sigma=1.25992 \AA$. It must be noted that the charges of both ions were uniformly scaled by a factor of 0.8 so as to accelerate the dynamics of the mixtures, which was shown necessary to have good agreement with the experimental results in LiTFSI WiS ${ }^{38}$.

In order to reach proper density, each system was firstly equilibrated at $298.15 \mathrm{~K}$ and 1 bar for 4 ns in the NpT ensemble by using Nosé-Hoover thermostat and barostat ${ }^{39-41}$ with relaxation times of 10 and 500 femtoseconds, respectively. The results are included in Table I and they show good agreement with the available experimental data. Then we performed a second equilibration of 60 ns within an NVT ensemble, followed by a production run of around $90 \mathrm{~ns}$ (with a time step $d t=1 \mathrm{fs}$ ) that was used to obtain structural and dynamic information about the systems. 


\section{RESULTS AND DISCUSSION}

Among all the mixtures considered in this article, the only one for which there is available experimental data concerning the solubility limit is $\mathrm{LiTFSI}_{2} \mathrm{H}_{2} \mathrm{O}$, whose value was reported to be a molality of $31.3 \mathrm{~m}$ at $303.15 \mathrm{~K}^{20}$. Accurately computing solubilities is very challenging in molecular dynamics ${ }^{42}$, so that we decided to analyze the medium/long range structure as a proxy to determine the relative miscibility of the systems. To do this, partial structure factors were computed using the formalism proposed by Faber and Ziman ${ }^{43}$, in which the structure factor is represented by the correlations between the different chemical species $\alpha$ and $\beta$ :

$$
S_{\alpha \beta}(q)=1+4 \pi \rho \int_{0}^{\infty} d r \frac{\sin (q r)}{q r} r^{2}\left(g_{\alpha \beta}(r)-1\right)
$$

where $g_{\alpha \beta}(r)$ are the partial radial distribution functions (RDF), which can be defined as

$$
g_{\alpha \beta}(r)=\frac{1}{\rho N}\left\langle\sum_{\alpha \beta} \delta\left(r-r_{\alpha \beta}\right)\right\rangle
$$

where $N$ is the number of particles in the system, $\rho$ stands for its number density, and brackets $(\langle\ldots\rangle)$ indicate an ensemble average.

Figure 2 shows the partial structure factors of (a) S-S and (b) $\mathrm{O}\left(\mathrm{H}_{2} \mathrm{O}\right)-\mathrm{O}\left(\mathrm{H}_{2} \mathrm{O}\right)$ atoms in the different mixtures. A demixing effect indicated by the peak at $q \rightarrow 0$ in the system based on the NF-anion can be seen. This is also shown by a snapshot of the simulation box included in Figure 2, in which we can clearly observe a segregated distribution of the liquid into (c) apolar (composed of the $\mathrm{CF}_{2^{-}}$and $\mathrm{CF}_{3^{-}}$groups of the $\mathrm{NF}$-anion) and (d) polar (consisting of lithium atoms, water molecules and the negatively charged $\mathrm{SO}_{3}$-group of the NF-anion) domains. That is, we can conclude that LiNF solubility in water is not high enough to satisfy the WiS condition and it will not be included in our analysis hereinafter. All the

other simulated WiS show similar structures, i.e. the formation of nano-heterogeneities ${ }^{17}$ at medium range as can be seen from the presence of intense peaks at $q$ values ranging from 0.95 to $1.5 \AA^{-1}$ and from 0.7 to $0.9 \AA^{-1}$ for the S-S and the O-O partial structure factors, respectively.

The use of WiS as electrolytes in energy storage devices will then be a compromise between a high concentration to extend the electrochemical stability window and a good ionic 


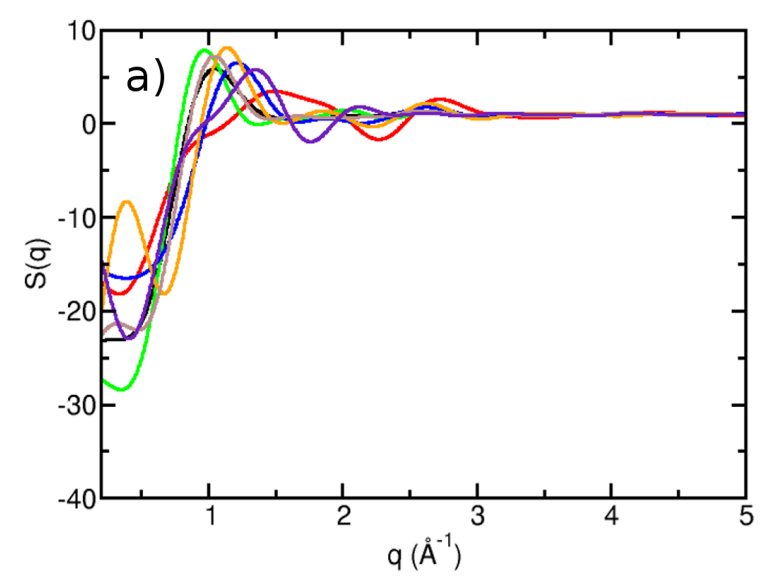

c)

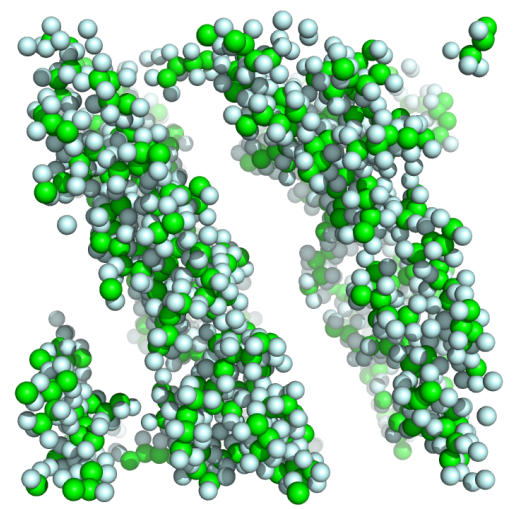

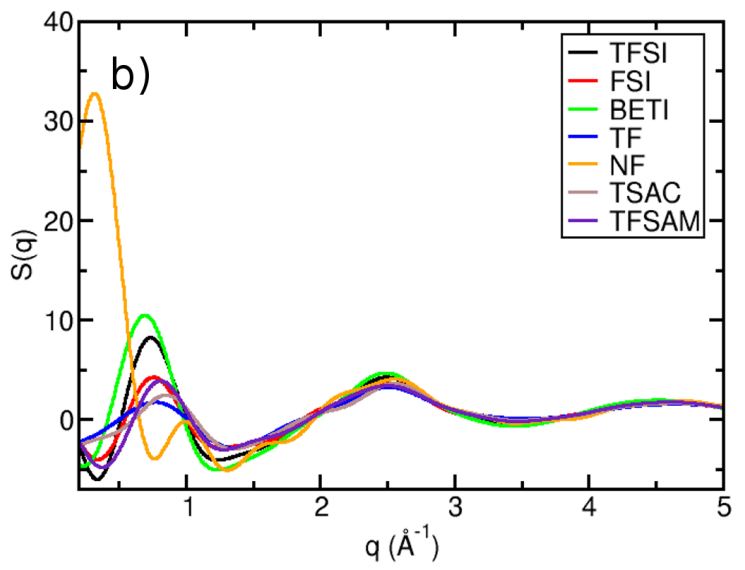

d)

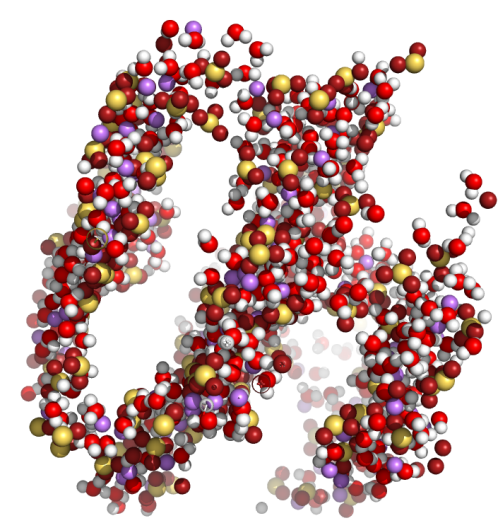

FIG. 2. Partial structure factors of (a) S-S and (b) $\mathrm{O}\left(\mathrm{H}_{2} \mathrm{O}\right)-\mathrm{O}\left(\mathrm{H}_{2} \mathrm{O}\right)$ atoms from MD simulations at $298.15 \mathrm{~K}$ for all the different mixtures. Snapshots of the simulation box that shows a segregation of the LiNF- $\mathrm{H}_{2} \mathrm{O}$ system into (c) apolar (composed of $\mathrm{C}$ and $\mathrm{F}$ atoms, in green and light blue, respectively) and (d) polar (consisting of lithium atoms in violet, $\mathrm{H}_{2} \mathrm{O}$ molecules in white and light red, and $\mathrm{S}$ and $\mathrm{O}$ atoms, in yellow and dark red, respectively) domains.

conductivity that guarantees acceptable power density ${ }^{9,20,21,44}$. Thus, the tailored design of superconcentrated aqueous electrolyte systems requires a deep understanding of the ion transport mechanism, and several reports suggested a fast Li ion transport through waterrich domains ${ }^{17,18}$. The two key collective transport quantities are the electrical conductivity and the viscosity. The former can be calculated in molecular dynamics simulations using

$$
\sigma=\frac{e^{2}}{k_{B} T V} \lim _{t \rightarrow \infty} \frac{1}{6 t}\left\langle\left|\sum_{i} q_{i} \Delta_{i}(t)\right|^{2}\right\rangle
$$

where $e$ is the elementary charge, $V$ is the volume of the simulation cell, $T$ is the temperature, 

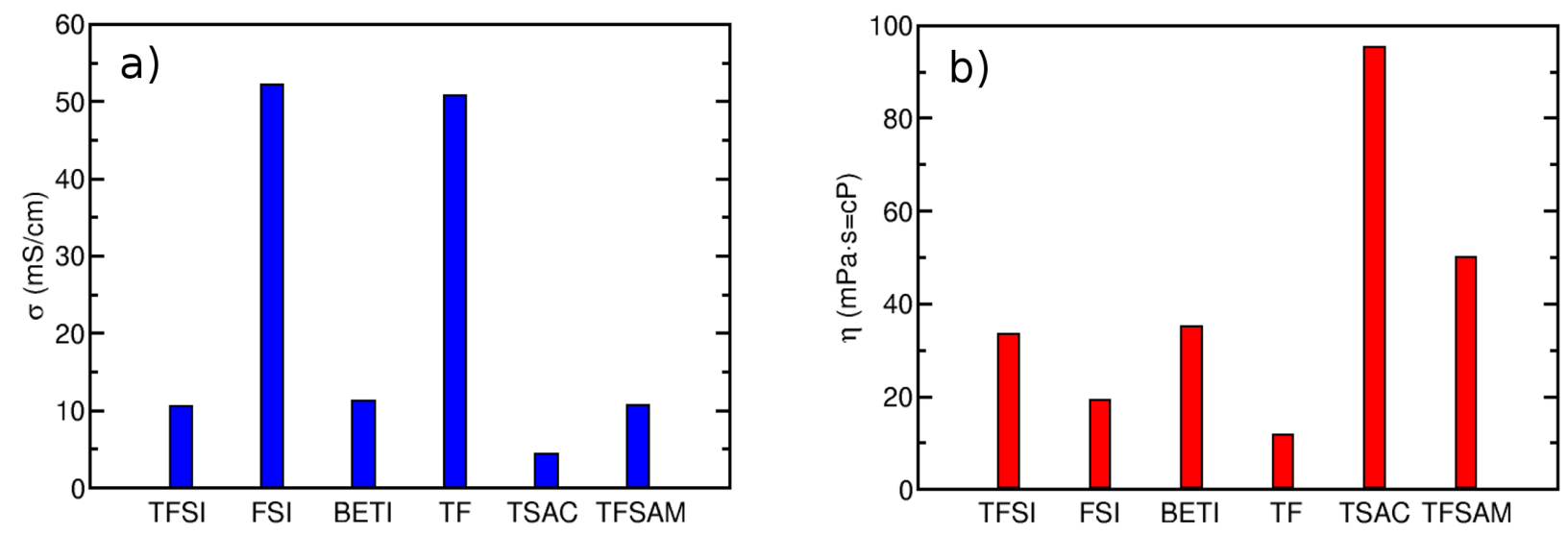

FIG. 3. Comparison of the conductivities (a) and the viscosities (b) in the different mixtures from MD simulations at $298.15 \mathrm{~K}$.

$k_{B}$ is the Boltzmann constant and $\Delta_{i}(t)=\vec{r}_{i}(t)-\vec{r}_{i}(0)$ is the displacement of the ion $i$, which carries a charge $q_{i}$, over a time interval $t$. This expression includes the contribution not only of the self-terms of each ion but also the complex effect of cross-correlations due to the correlated motion of ions. The electrical conductivities obtained using eq.(3) are included in Figure 3.a.

In addition, the shear viscosity was computed within the Green-Kubo (GK) formalism ${ }^{45}$ by integration of the stress-tensor correlation function using

$$
\eta=\frac{V}{k_{B} T} \int_{0}^{\infty}\left\langle\Pi_{\alpha \beta}(0) \Pi_{\alpha \beta}(t)\right\rangle d t
$$

where $\Pi_{\alpha \beta}$ represents any of the five independent components of the stress tensor, $\Pi_{x y}, \Pi_{x z}$, $\Pi_{y z}, \Pi_{x x-y y}, \Pi_{2 z z-x x-y y}$. The values obtained for the viscosity of each system, that is, the value of the plateau at which the running integral in eq.(4) converges after a certain time, are shown in Figure 3.b.

The simulations predict a $\mathrm{LiTFSI}-\mathrm{H}_{2} \mathrm{O}$ viscosity in good agreement with experimental data $\left(\approx 33 \mathrm{cP}\right.$ instead of $22 \mathrm{cP}^{38}$ ), while they underestimate the ionic conductivity by a factor $2^{8,20,38}$, which corresponds to typical error for the prediction of transport properties in electrolytes by non-polarizable molecular dynamics. Although the use of a different parameterization for the partial charges and Lennard-Jones parameters of the anion could improve the situation ${ }^{38}$, the present parameters were chosen in order to keep consistency between all the different anions studied and to compare them without introducing any bias. 
When comparing the various liquids, we observe that they can be separated between three groups. Firstly, the superconcentrated $\mathrm{LiFSI}-\mathrm{H}_{2} \mathrm{O}$ and $\mathrm{LiTF}_{2} \mathrm{H}_{2} \mathrm{O}$ mixtures have low viscosities and high ionic conductivities. In particular, the latter $(\sim 50 \mathrm{mS} / \mathrm{cm}$ in both cases $)$ is greater than the values observed in typical non-aqueous electrolytes used in commercial Li-ion batteries ${ }^{46}$ and supercapacitors ${ }^{47}$. Then we observe that the LiBETI- $\mathrm{H}_{2} \mathrm{O}$ mixture shows similar performances as the reference TFSI system. The LiTFSAM- $\mathrm{H}_{2} \mathrm{O}$ has a larger viscosity but a simular conductivity as those two, so it can be put in the same group. Finally, the system involving the TSAC anions shows significantly poorer performances than all the others, with a viscosity reaching $\sim 100 \mathrm{cP}$ and a low ionic conductivity. It is worth noting that the variations between anions cannot easily be explained using their relative size/weights since for example TFSAM and FSI have relatively similar sizes, as well as TFSI and TSAC, while BETI has the largest fluorocarbonated chains. Nevertheless, if we split them between the symmetric (FSI, TFSI and BETI by order of increasing size) and asymmetric (TF, TFSAM and TSAC) ones, we observe a trend for the viscosity to increase with the anion size. Based on this observation, the best transport properties seem to arise for small symmetric anions.

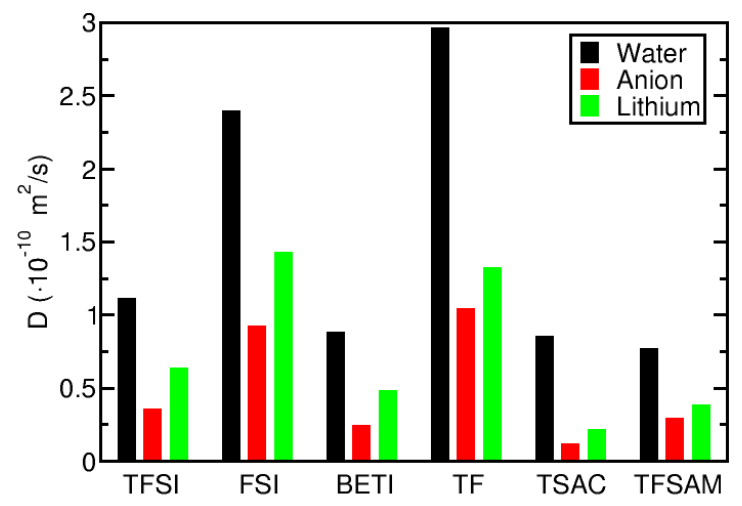

FIG. 4. Comparison of the self-diffusion coefficients of the water molecules, the various organic anions and the lithium cations in the different mixtures from MD simulations at $298.15 \mathrm{~K}$.

In order to analyze further the individual dynamics of the liquids, the self-diffusion coefficient of the species $i$ can be calculated from the long-time limit of the mean-squared displacement (MSD) by using 


$$
D_{i}=\lim _{t \rightarrow \infty} \frac{1}{6 t}\left\langle\left|\Delta_{i}(t)\right|^{2}\right\rangle
$$

Note that a correction due to the use of periodic boundary conditions must be added ${ }^{48}$. Figure 4 shows the values obtained for $\mathrm{H}_{2} \mathrm{O}$ molecules, lithium cations and the various anions. In agreement with previous works ${ }^{17,38}$, it can be seen that water molecules are the most mobile species, followed by lithium cations and anions. This corresponds to an opposite behaviour with respect to the dynamics of the ions in typical ionic liquid electrolytes, where anions generally diffuse faster than cations ${ }^{17,44,49}$.

The individual dynamics provide further points of comparison between the various electrolytes. The main difference with respect to the analysis of the collective transport properties is that the diffusivity of water molecules in the TSAC-based system is similar to the cases of TFSI, BETI and TFSAM. This points towards a predominant role of the low diffusivity of the anions in the high viscosity of the system. We also observe that the lithium ion dynamics is enhanced in LiTFSI- $\mathrm{H}_{2} \mathrm{O}$ with respect to BETI and TFSAM-based systems, which points towards a better performance of the former in Li-ion batteries applications despite the similar conductivities of the systems.

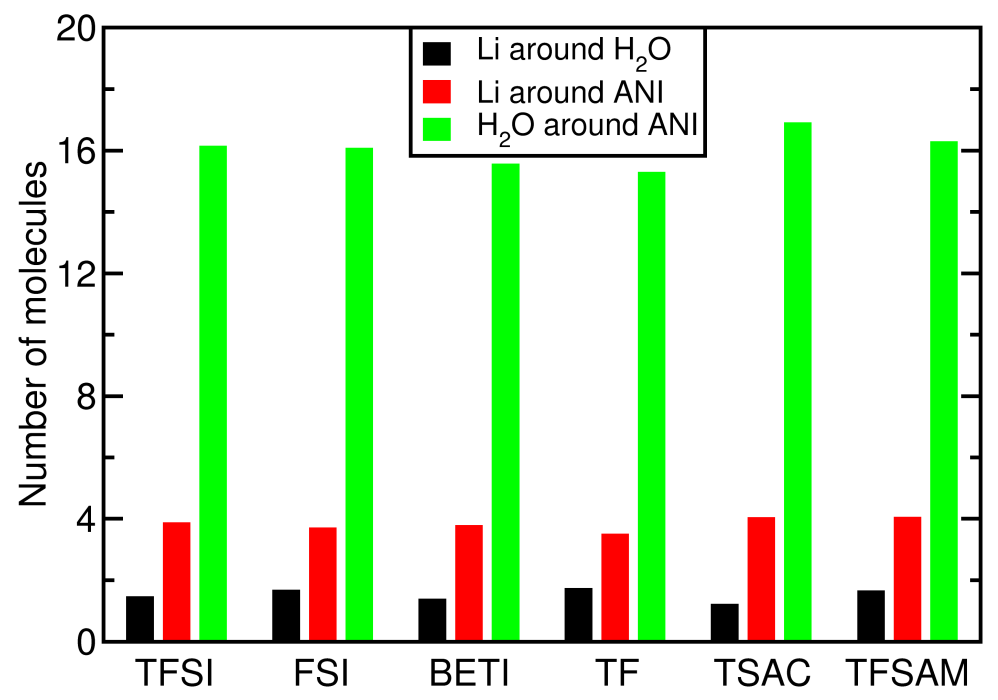

FIG. 5. Neighbour count around the reference subset from Voronoi analysis for the different mixtures from MD simulations. 
Unlike in ionic liquid/water systems, where the interaction between $\mathrm{H}_{2} \mathrm{O}$ molecules and anions is generally stronger than with the cations ${ }^{50}$, in WiS electrolytes they are strongly attracted by the lithium ions ${ }^{8}$. At infinite dilution the first solvation shell of $\mathrm{Li}^{+}$is made of four water molecules. At the molality studied here, the ratio $\mathrm{H}_{2} \mathrm{O} / \mathrm{Li}$ is smaller than four. As a consequence the first solvation shell of lithium cations includes both water and anions ${ }^{16,22}$. It was previously reported that as the concentration of the mixture increases the coordination environment is expected to change from solvent-separated ion pairs (SSIP) to a majority of contact ion pairs (CIPs) and ionic aggregates (AGGs) $)^{17,23,51}$. In the present work, we employed the trajectory analysis program TRAVIS ${ }^{52}$ to analyze the formation of domains in these electrolytes, whose methodology is based on Voronoi tessellation ${ }^{53,54}$. The subsets were defined so as to match the three types of molecules in the mixtures, and two subsets were considered to be neighbours if they share at least a common face. This approach provided us with valuable information on the neighborhood of the reference subset, and the results are included in Figure 5. We can observe that the choice of the anion does not have a remarkable impact on the number of molecules neighbouring a reference one, so that the WiS studied here have qualitatively similar structures.

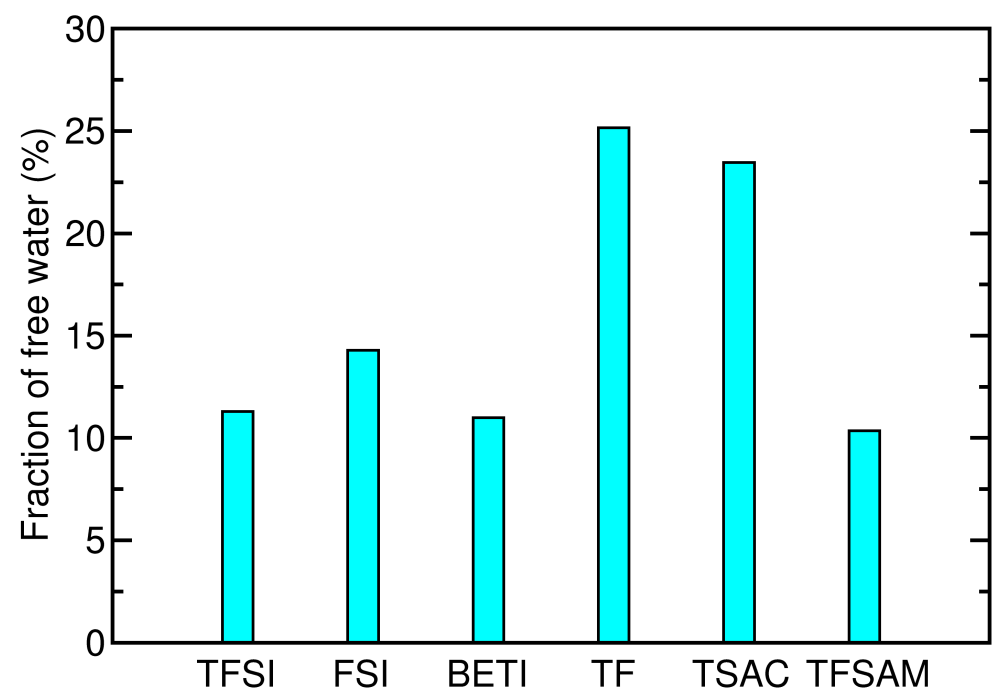

FIG. 6. The fraction of free water not bound to any Li cation obtained for the different mixtures from MD simulations. 
Nevertheless, the small variations observed for the coordination of the Li ions by the water molecules may lead to substantial changes in the concentration of "free" water molecules at such molalities. A water molecule is considered to be free when it is not coordinated to any lithium ion, but it is worth noting that in WiS they remain partly coordinated to other water molecules through an extended hydrogen-bond network, which will affect their reactivity as well ${ }^{55}$. The amount of free water molecules is shown in Figure 6 . We observe that it is significantly higher in the TF and the TSAC-based systems. This probably affects significantly the electrochemical window because non-coordinated water molecules can adsorb more easily on the positive electrode than the lithium-coordinated ones (due to the Coulombic repulsion of the $\mathrm{Li}^{+}$ions). At the negative electrode it is also expected that the formation of the protective SEI will be more difficult for these salts.

\section{CONCLUSIONS}

In conclusion, we have studied through extended molecular dynamics simulations a series of WiS electrolytes where the nature of the anion was systematically changed. The same force field was used for all the simulations and the molality was kept fixed at $15 \mathrm{~m}$ in order to allow for representative comparisons. By analyzing the structure factors, we observed that the NF anion has a strong tendency to demix from the aqueous phase due to its highly hydrophobic fluorinated chain. All the other anions have structural features typical of water-in-salts, namely the formation of nanoheterogeneities with two type of domains, the first ones containing mostly the anions and the second ones made of solvated lithium ions linked through the hydrogen-bond network of the water molecules.

In a second step, by computing the transport properties we have identified that they do not vary in a systematic way with the size or the molecular weight of the anion. For a given ion size, the use of symmetric anions leads to a lower viscosity and increased ionic conductivities and diffusion coefficients. The TF and FSI display higher diffusion coefficients, followed by the TFSI, BETI and TFSAM-based systems. Finally, the TSAC has a poor viscosity and should therefore be avoided in electrochemical systems.

Finally, we have looked at the speciation of the systems, we have observed that the TF-based system has a relatively high amount of free water molecules, which may affect its electrochemical window. Nevertheless, feature work should adress more specifically the 
reactivity of the molecules since the mechanisms that have been identified for TFSI may not be adequate for all the other anions. This will require the use of density functional theory-based simulations in order to allow the formation/break of chemical bonds.

\section{DATA AVAILABILITY}

The raw/processed data required to reproduce these findings are available to download from https://gitlab.com/ampere2

\section{ACKNOWLEDGMENTS}

This work was supported by the French National Research Agency (Labex STORE-EX, Grant No. ANR-10-LABX-0076). This project has received funding from the European Research Council (ERC) under the European Union's Horizon 2020 research and innovation programme (grant agreement no. 771294).We acknowledge support from EoCoE, a project funded by the European Union Contract No. H2020-EINFRA-2015-1-676629, from the DSM-Energie programme of CEA and from the Eurotalent programme. This work was granted access to the HPC resources of CINES under the allocation A0080910463 made by GENCI.

\section{REFERENCES}

${ }^{1}$ G. E. Blomgren, J. Electrochem. Soc. 164, A5019 (2017).

${ }^{2}$ M. Armand and J. M. Tarascon, Nature 451, 652 (2008).

${ }^{3}$ C. P. Grey and J. M. Tarascon, Nature 16, 45 (2016).

${ }^{4}$ A. Hammami, N. Raymond, and M. Armand, Nature 424, 635 (2003).

${ }^{5}$ L. Xia, L. Yu, D. Hu, and G. Z. Chen, Mat. Chem. Front. 1, 584 (2017).

${ }^{6}$ Y. Wang, J. Yi, and Y. Xia, Adv. Energy Mater. 2, 830 (2012).

${ }^{7}$ C. Wessells, R. A. Huggins, and Y. Cui, J. Power Sources 196, 2884 (2011).

${ }^{8}$ L. Suo, O. Borodin, T. Gao, M. Olguin, J. Ho, X. Fan, C. Luo, C. Wang, and K. Xu, Science 350, 938 (2015).

${ }^{9}$ L. Suo, Y.-S. Hu, H. Li, M. Armand, and L. Chen, Nat. Commun. 4, 1 (2013).

${ }^{10}$ O. Borodin, J. Self, K. A. Persson, C. Wang, and K. Xu, Joule 4, 69 (2020). 
${ }^{11}$ Z. Li, G. Jeanmairet, T. Mendez-Morales, B. Rotenberg, and M. Salanne, J. Phys. Chem. C 122, 23917 (2018).

${ }^{12}$ N. Dubouis, P. Lemaire, B. Mirvaux, E. S. ad M. Deschamps, and A. Grimaud, Energy Environ. Sci. 11, 3491 (2018).

${ }^{13}$ R. Bouchal, Z. Li, C. Bongu, S. Le Vot, R. Berthelot, B. Rotenberg, F. Favier, S. Freunberger, M. Salanne, and O. Fontaine, Angew. Chem., Int. Ed. 59, 15913 (2020).

${ }^{14}$ H.-G. Steinrück, C. Cao, M. Lukatskaya, C. Takacs, G. Wan, D. Mackanic, Y. Tsao, J. Zhao, B. Helms, K. Xu, et al., Angew. Chem., Int. Ed. in press, 10.1002/ange.202007745 (2020).

${ }^{15}$ L. Suo, F. Han, X. Fan, H. Liu, K. Xu, and C. Wang, J. Mater. Chem. A 4, 6639 (2016).

${ }^{16}$ R. S. Kuhnel, D. Reber, A. Remhof, R. Figi, D. Bleiner, and C. Battaglia, Chem. Commun. 52, 10435 (2016).

${ }^{17}$ O. Borodin, L. Suo, M. Gobet, X. Ren, F. Wang, A. Faraone, J. Peng, M. Olguin, M. Schroeder, M. S. Ding, et al., ACS Nano 11, 10462 (2017).

${ }^{18}$ J. Lim, K. Park, H. Lee, J. Kim, K. Kwak, and M. Cho, J. Am. Chem. Soc. 140, 15661 (2018).

${ }^{19}$ L. Coustan, G. Shul, and D. Bélanger, Electrochem. Commun. 77, 89 (2017).

${ }^{20}$ P. Lannelongue, R. Bouchal, E. Mourad, C. Bodin, M. Olarte, S. le Vot, F. Favier, and O. Fontaine, J. Electrochem. Soc. 165, A657 (2018).

${ }^{21}$ Q. Dou, S. Lei, D.-W. W., Q. Zhang, D. Xiao, H. Guo, A. Wang, H. Yang, Y. Li, S. Shi, et al., Energy Environ. Sci. 11, 3212 (2018).

${ }^{22}$ M. R. Lukatskaya, J. I. Feldblyum, D. G. Mackanic, F. Lissel, D. L. Michels, Y. Cui, and Z. Bao, Energy Environ. Sci. 11, 2876 (2018).

${ }^{23}$ L. Suo, O. Borodin, Y. Wang, X. Rong, W. Sun, X. Fan, S. Xu, M. A. Schroeder, A. V. Cresce, F. Wang, et al., Adv. Energy Mater. 7, 1701189(1) (2017).

${ }^{24}$ R. S. Kuhnel, D. Reber, and C. Battaglia, ACS Energy Lett. 2, 2005 (2017).

${ }^{25}$ D. Reber, R. S. Kuhnel, and C. Battaglia, Sustainable Energy Fuels 1, 2155 (2017).

${ }^{26}$ J. Han, H. Zhang, A. Varzi, and S. Passerini, ChemSusChem 11, 3704 (2018).

${ }^{27}$ L. Ma, M. A. Schroeder, O. Borodin, T. P. Pollard, M. S. Ding, C. Wang, and K. Xu, Nat. Energy in press, 10.1038/s41560-020-0674-x (2020).

${ }^{28}$ L. Suo, O. Borodin, W. Sun, X. Fan, C. Yang, F. Wang, T. Gao, Z. Ma, M. Schroeder, A. von W. Cresce, et al., Angew. Chem. Int. Ed. 55, 7136 (2016). 
${ }^{29}$ J. Vatamanu and O. Borodin, J. Phys. Chem. Lett. 8, 4362 (2017).

${ }^{30}$ N. Dubouis, C. Park, M. Deschamps, S. Abdelghani-Idrissi, M. Kanduc, A. Colin, M. Salanne, J. Dzubiella, A. Grimaud, and B. Rotenberg, ACS Cent. Sci. 5, 640 (2019).

${ }^{31}$ C. Yang, J. Chen, X. Ji, T. P. Pollard, X. Lü, C.-J. Sun, S. Hou, Q. Liu, C. Liu, T. Qing, et al., Nature 569, 245 (2019).

${ }^{32}$ M. R. Lukatskaya, J. I. Feldblyum, D. G. Mackanic, F. Lissel, D. L. Michels, Y. Cui, and Z. Bao, Energy Environ. Sci. 11, 2876 (2018).

${ }^{33}$ S. Plimpton, J. Comp. Phys. 117, 1 (1995).

${ }^{34}$ L. Martínez, R. Andrade, E. G. Birgin, and J. M. Martínez, J. Comput. Chem. 30, 2157 (2009).

${ }^{35}$ H. J. C. Berendsen, J. R. Grigera, and T. P. Straatsma, J. Phys. Chem. 91, 6269 (1987.).

${ }^{36}$ J. N. C. Lopes, J. Deschamps, and A. A. H. Pádua, J. Phys. Chem. B 108, 2038 (2004).

${ }^{37}$ A. S. L. Gouveia, C. E. S. Bernardes, L. C. Tomé, E. Lozinskaya, Y. Vygodskii, A. S. Shaplov, J. N. C. Lopes, and I. M. Marrucho, Phys. Chem. Chem. Phys. 19, 29617 (2017).

${ }^{38}$ Z. Li, R. Bouchal, T. Mendez-Morales, A.-L. Rollet, C. Rizzi, S. Le Vot, F. Favier, B. Rotenberg, O. Borodin, O. Fontaine, et al., J. Phys. Chem. B 123, 10514 (2019).

${ }^{39}$ W. G. Hoover, Phys. Rev. A 31, 1695 (1985).

${ }^{40}$ S. Melchionna, G. Ciccotti, and B. L. Holian, Mol. Phys. 78, 533 (1993).

${ }^{41}$ G. J. Martyna, M. L. Klein, and M. Tuckerman, J. Chem. Phys. 97, 2635 (1992).

${ }^{42}$ M. Ferrario, G. Ciccotti, E. Spohr, T. Cartailler, and P. Turq, J. Chem. Phys. 117, 4947 (2002).

${ }^{43}$ T. E. Faber and J. M. Ziman, Phil. Mag. 11, 153 (1965).

${ }^{44}$ R. Yang, Y. Zhang, K. Takechi, and E. J. Maginn, J. Phys. Chem. C 122, 13815 (2018).

${ }^{45}$ M. P. Allen and D. J. Tildesley, Computer simulations of liquids (Oxford University Press, 1987).

${ }^{46}$ K. Xu, Chem. Rev. 104, 4303 (2004).

${ }^{47}$ Z. Lin, E. Goikolea, A. Balducci, K. Naoi, P.-L. Taberna, M. Salanne, G. Yushin, and P. Simon, Mater. Today 21, 419 (2018).

${ }^{48}$ I.-C. Yeh and G. Hummer, J. Phys. Chem. B 108, 15873 (2004).

${ }^{49}$ H. Liu and E. Maginn, J. Chem. Phys. 139, 114508 (2013).

${ }^{50}$ B. Docampo-Álvarez, V. Gómez-González, H. Montes-Campos, J. M. Otero-Mato, T. Méndez-Morales, O. Cabeza, L. J. Gallego, R. M. Lynden-Bell, V. B. Ivanistsev, M. V. 
Fedorov, et al., J. Phys.: Condens. Matter 28, 464001(1) (2016).

${ }^{51}$ J. Wang, Y. Yamada, K. Sodeyama, C. H. Chiang, Y. Tateyama, and A. Yamada, Nat. Commun. 7, 1 (2016).

${ }^{52}$ M. Brehm and B. Kirchner, J. Chem. Inf. Model. 51, 2007 (2011).

${ }^{53}$ M. Brehm, H. Weber, M. Thomas, O. Hollóczki, and B. Kirchner, Chem. Phys. Chem. 16, 3271 (2015).

${ }^{54}$ E. Elfgen, O. Hollóczki, and B. Kirchner, Acc. Chem. Res. 50, 2949 (2017).

${ }^{55}$ N. Dubouis, A. Serva, R. Berthin, G. Jeanmairet, B. Porcheron, E. Salager, M. Salanne, and A. Grimaud, Nat. Catal. 3, 656 (2020). 


\section{GRAPHICAL ABSTRACT}
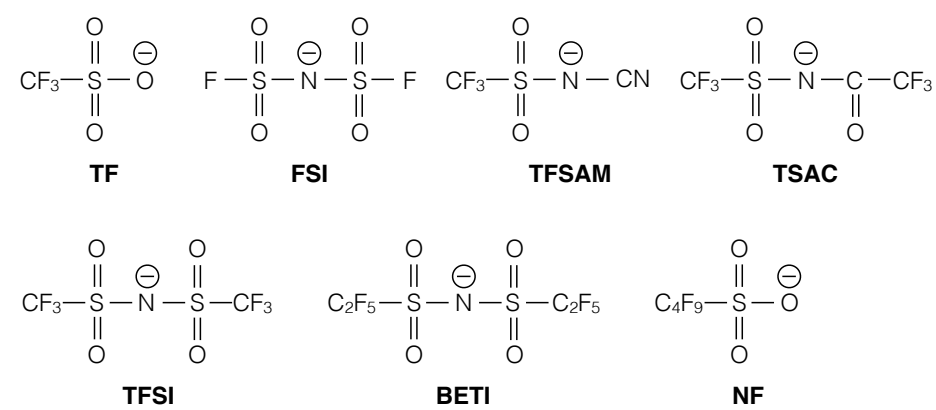

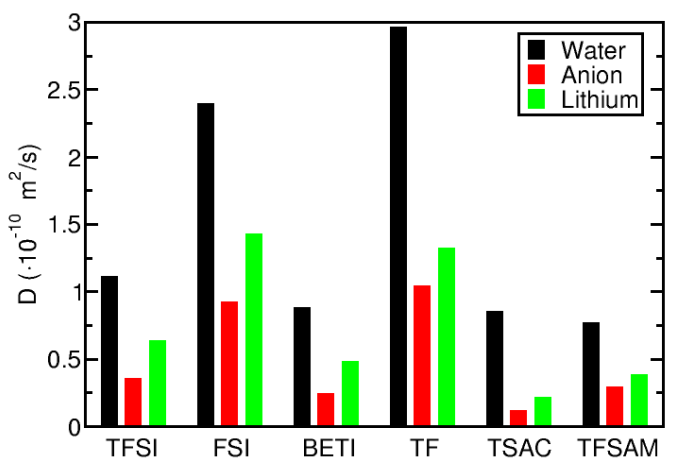

\section{KEYWORDS}

Superconcentrated electrolytes; Supercapacitors; Li-ion batteries; Transport properties 